Pacific Journal of Mathematics

ONE PARAMETER GROUPS OF ISOMETRIES ON CERTAIN

SNACK SPACe 


\section{ONE PARAMETER GROUPS OF ISOMETRIES ON CERTAIN BANACH SPACES}

\section{Richard J. Fleming, Jerome A. Goldstein and James E. Jamison}

Banach spaces of class $\mathscr{S}$ were introduced by Fleming and Jamison. This broad class includes all Banach spaces having hyperorthogonal Schauder bases and, in particular, $\mathscr{S}$ includes all Orlicz spaces $L^{\Phi}$ on an atomic measure space such that the characteristic functions of the atoms form a basis for $L^{\Phi}$. The main theorem gives the structure of one parameter strongly continuous (or $\left(C_{0}\right)$ ) groups of isometries on Banach spaces of class $\mathscr{S}$. Other results correct and complement the work of Goldstein on groups of isometries on Orlicz spaces over atomic measure spaces.

\section{In $[4$, p. 390] the following theorem was stated.}

THEOREM 1. Let $X=L^{\Phi}(\Omega, \Sigma, \mu)$ be an Orlicz space on an atomic measure space. Let $T=\left\{T^{t} \mid t \in \mathbf{R}=(-\infty, \infty)\right\}$ be a $\left(C_{0}\right)$ group of isometries on $X$ with infinitesimal generator $A$, and suppose $(*)$ and $(* *)$ hold. ${ }^{1}$ Suppose $X$ is not a Hilbert space, i.e. $\Phi(s)$ is not of the form $\Phi(s) \equiv$ const $\times s^{2}$.

(I) If $X$ is a real space, then $T^{t}=I$ for each $t \in \mathbf{R}$.

(II) If $X$ is a complex space, then there exists a function $g: \Omega \rightarrow \mathbf{R}$ such that $\left(T^{t} f\right)(\omega)=\exp \{\operatorname{itg}(\omega)\} f(\omega)$ for each $f \in X$ and each $\omega \in \Omega$.

The idea of the proof in [4] is as follows. Write $\Omega=\left\{\omega_{i}\right\}$ and view $A$ as a matrix whose ij entry is $A\left(\delta\left(\omega_{i}\right)\right)\left(\omega_{i}\right)$, where $\delta\left(\omega_{i}\right)$ is the function which is 1 at $\omega_{i}$ and 0 everywhere else. $A$ is a diagonal matrix if and only if $T$ satisfies (I) or (II). It was assumed that $A$ was not diagonal, and the proof given in [4] established the existence of a $\tau, 0<\tau \leqq \infty$, such that

$$
\Phi(s)=\text { const } \times s^{2} \text { for } 0 \leqq s<\tau \text {. }
$$

It was asserted in [4] that $\tau=\infty$, which means that $X$ is a Hilbert space. But in fact this is not correct as the following example shows.

${ }^{1}(*)$ and $(* *)$ are mild technical conditions on $\Phi$ and $A$. (Cf. [1, p. 389] for the precise statement.) $X$ cannot be an infinite dimensional $L^{\infty}$ space, but otherwise $(*)$ and $(* *)$ are not very restrictive. 
EXAMPLE 1. Let $(\Omega, \Sigma, \mu)$ be an atomic measure space defined as follows. $\Omega$ consists of three points $\omega_{1}, \omega_{2}, \omega_{3}$, and $\mu\left(\omega_{1}\right)=\mu\left(\omega_{2}\right)=1 / 2$, $\mu\left(\omega_{3}\right)=1 / 200$. Let $\Phi$ and $\Psi$ be defined as follows:

$$
\begin{aligned}
& \Phi(s)= \begin{cases}s^{2} / 2, & s \in[0,4] \\
\left(s^{3}+32\right) / 12, & s \in[4, \infty),\end{cases} \\
& \Psi(s)= \begin{cases}s^{2} / 2, & s \in[0,4] \\
\left(4 s^{3 / 2}-8\right) / 3, & s \in[4, \infty) .\end{cases}
\end{aligned}
$$

Since $\Phi^{\prime}$ and $\Psi^{\prime}$ are continuous and $\Phi(1)+\Psi(1)=1$, all the conditions of [4] are satisfied. If we define

$$
(H f)(\omega)= \begin{cases}f\left(\omega_{2}\right), & \omega=\omega_{1} \\ f\left(\omega_{1}\right), & \omega=\omega_{2} \\ 2 f\left(\omega_{3}\right), & \omega=\omega_{3},\end{cases}
$$

then $H$ is an Hermitian operator (in the sense of Lumer [5]) and $\left\{e^{i t H} \mid t \in \mathbf{R}\right\}$ is a strongly continuous group of isometries on the complex space $L^{\Phi}(\Omega, \Sigma, \mu)$. A little computation yields

$$
e^{u H}=\left(\begin{array}{ccc}
\cos t t & i \sin t & 0 \\
i \sin t & \cos t & 0 \\
0 & 0 & e^{2 t t}
\end{array}\right)
$$

where the matrix is given relative to the basis $\left\{\delta\left(\omega_{1}\right)\right\}_{j=1}^{3}$ consisting of the characteristic functions of the atoms. This group of isometries is clearly not of the form described in Theorem 1.

The correct version of the theorem is

TheOREM 2. Let $X=L^{\Phi}(\Omega, \Sigma, \mu)$ be an Orlicz space on an atomic measure space. Let $T=\left\{T^{t} \mid t \in \mathbf{R}\right\}$ be a $\left(C_{0}\right)$ group of isometries on $X$ with generator $A$, and suppose (*) and (**) hold. Then either there exists a $\tau$, $0<\tau \leqq \infty$, such that $\Phi(s)=c s^{2}$ for $0 \leqq s<\tau$, where $c$ is a positive constant, or else:

(I) If $X$ is a real space, $T^{t}=I$ for each $t \in \mathbf{R}$.

(II) If $X$ is a complex space, there exists a function $g: \Omega \rightarrow \mathbf{R}$ such that $\left(T^{t} f\right)(\omega)=\exp \{\operatorname{itg}(\omega)\} f(\omega)$ for each $f \in X$ and each $\omega \in \Omega$.

Remark 1. Let $T$ on $X$ be not of the form (I) or (II). Then the $\tau$ constructed in the proof of Theorem 1 [4, p. 391] is 


$$
\tau=\sup \Phi\left(|f(\omega)| /\|f\|_{\Phi}\right)
$$

the supremum being over the set of all functions $f=\alpha_{1} \delta\left(\omega_{1}\right)+\alpha_{2} \delta\left(\omega_{2}\right)$ where $\alpha_{1}, \alpha_{2}$ are nonzero scalars, $\delta\left(\omega_{i}\right)$ is as before the characteristic function of $\omega_{1}$, and $\omega_{1}, \omega_{2}$ are distinct members of $\Omega$ such that $A\left(\delta\left(\omega_{1}\right)\right)$ does not vanish at $\omega_{2}$. The existence of such a pair $\omega_{1}, \omega_{2}$ follows from the fact that $A$ is not a diagonal matrix since $T$ is not of the form (I) or (II).

Remark 2. Theorem 2 does not single out Hilbert space among the Orlicz spaces, as asserted in [4], but it does single out Hilbert spaces among a class of Orlicz spaces including the Lebesgue $L^{p}$ spaces (over atomic measure spaces).

The results of $\S 4$ of [4] which were asserted to be consequences of Theorem 1 are in fact consequences of Theorem 2, so they are correct as stated.

REMARK 3. Observe that the space in Example 1 can be written as a direct sum $L^{\Phi}(\Omega, \Sigma, \mu)=X_{1}+X_{2}$ where $X_{1}=\left\{f \mid f\left(\omega_{3}\right)=0\right\}$ and $X_{2}=$ $\left\{f \mid f\left(\omega_{i}\right)=0\right.$ for $\left.i=1,2\right\}$. Also note that the operators in the group can be written as

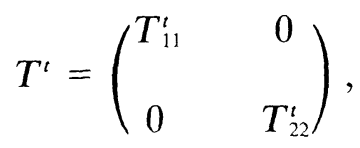

where $\left\{T_{j j}^{t} \mid t \in \mathbf{R}\right\}$ is a $\left(C_{0}\right)$ group of isometries on $X_{j}, j=1,2$. Furthermore, $X_{1}, X_{2}$ are two dimensional and one dimensional Hilbert spaces respectively.

It will follow from Example 5 and Theorem 3 below that any counterexample to Theorem 1 is essentially of the same nature as is Example 1.

2. We now consider a class of spaces which includes the Orlicz spaces $L^{\Phi}(\Omega, \Sigma, \mu)$ on atomic measure spaces, and we characterize the $\left(C_{0}\right)$ groups of isometries on spaces of this class.

Definition. A Banach space $(X, \nu)$ is said to be a member of class $\mathscr{S}$ if there exists a Banach space of sequences $(E, \mu)$ with absolute norm such that (i) $(E, \mu)$ possesses a sufficiently $l^{p}$-like semi inner product; (ii) there exists a sequence $\left\{X_{l}\right\}$ of (not necessarily separable) Hilbert spaces such that given $x \in X$ there exists a unique sequence $\left\{x_{i}\right\}\left(x_{i} \in X_{i}\right)$ for which $x=\sum_{t=1}^{n} x_{t}$ if $\left\{X_{t}\right\}$ is a finite sequence or $x=\lim _{n \rightarrow \infty} \sum_{i=1}^{n} x_{l}$ in the infinite case; (iii) if $x=\Sigma x_{\imath} \in X$, then $\left(\left\|x_{\imath}\right\|\right) \in E$ and $\nu(x)=\mu\left[\left(\left\|x_{i}\right\|\right)\right]$. In this case we write $X=\left\{X,\left\{X_{i}\right\}, E, \mu\right\}$. 
The exact meaning of a sufficiently $l^{p}$-like semi inner product will not be used explicitly in what follows and therefore will not be given. (See [2] for the definition.) Rather, we will give several examples of spaces of class $\mathscr{S}$. As the examples will show, the class $\mathscr{S}$ is quite broad. In fact, $\mathscr{I}$ contains all Banach spaces which have hyperorthogonal Schauder bases [2].

EXAmple 2. Let $1 \leqq p<\infty$ and let $\left\{X_{l}\right\}$ be a sequence of (not necessarily separable) Hilbert spaces. Let $l^{p}\left(X_{\imath}\right)=\left\{\left(x_{\kappa}\right) \mid x_{\kappa} \in X_{\kappa}\right.$, $\left.\Sigma\left\|x_{\kappa}\right\|^{p}<\infty\right\}$ and let $\nu\left[\left(x_{k}\right)\right]=\left(\Sigma\left\|x_{k}\right\|^{p}\right)^{1 / p}$. This space is a member of class $\mathscr{S}$ and the associated sequence space $(E, \mu)$ is just $\left(l^{p},\|\cdot\|_{p}\right)$. Since the $\left\{X_{i}\right\}$ can all be one dimensional, $l^{p} \in \mathscr{Y}$.

EXAmPle 3. Let $1 \leqq p<\infty$. The Lorentz sequence spaces $d(a, p)$ [1] are members of class $\mathscr{S}$. They are defined as follows: For any $a=\left(a_{\imath}\right) \in c_{0} \mid l^{1}, a_{1} \geqq a_{2} \geqq a_{3} \geqq \cdots>0$,

$$
d(a, p)=\left\{\left(x_{\kappa}\right) \in c_{0}: \sup _{\sigma \in \pi} \sum_{i=1}^{\infty}\left|x_{\sigma(t)}\right|^{p} a_{t}<\infty\right\}
$$

where $\pi$ is the set of all permutations of the natural numbers. The linear space $d(a, p)$ becomes a Banach space if endowed with the norm $\nu(x)=\left[\sup _{\sigma \in \pi} \sum_{i=1}^{\infty}\left|x_{\sigma(l)}\right|^{p} a_{i}\right]^{1 / p}$. For this space it can be shown that all of the $X_{\iota}$ are just the one dimensional spaces spanned by the basis elements $e_{\imath}$ and $(E, \mu)=(d(a, p), \nu)$.

EXAMPLE 4. Let $\left(p_{t}\right)$ denote a sequence of real numbers with $1<b \leqq p_{\imath} \leqq c<\infty$ for all $i$. If $x=\left(x_{t}\right)$ is a sequence of complex numbers, let $M(x)=\sum_{t=1}^{\infty}\left|x_{\imath}\right|^{p_{t}} / p_{i}$. Now let $l\left(p_{t}\right)=\left\{\left(x_{i}\right) \mid M(\lambda x)<\infty\right.$ for some $\lambda>0\}$ and define $\nu(x)=\inf \{1 / \epsilon \mid M(\epsilon x) \leqq 1\}$. Under these hypotheses the space is a uniformly convex Banach space [7] and is a member of class $\mathscr{S}$. It can be shown that in this case all of the $X_{i}$ 's are one dimensional except possibly one which is the $\overline{\operatorname{span}}\left\{e_{1} \mid p_{t}=2\right\}$, where $e_{t}$ denotes the element which is 1 in the $i$ th coordinate and zero otherwise. The associated sequence space $(E, \mu)$ is $l\left(\hat{p}_{\imath}\right)$ where $\hat{p}_{t}=2$ for at most one $i$ and $\hat{p}_{\imath}=p_{\imath}$ for $p_{\imath} \neq 2$.

EXAmple 5. Let $(\Omega, \Sigma, \mu)$ be a purely atomic measure space and let $L^{\Phi}(\Omega, \Sigma, \mu)$ be an Orlicz space over it. If the characteristic functions of the atoms form a basis for $L^{\Phi}(\Omega, \Sigma, \mu)$, then $L^{\Phi}(\Omega, \Sigma, \mu) \in \mathscr{S}$. In this case, the $X_{t}$ 's are all one dimensional except possibly one. This can occur whenever $\Phi(s)=c s^{2}$ for $s \in[0, \tau]$, and in this case it is given by $\overline{\operatorname{span}}\left\{\delta\left(\omega_{i}\right) \mid \tau \geqq\left(c \mu\left(\omega_{\imath}\right)\right)^{-\frac{1}{2}}\right\}$, where $\delta\left(\omega_{\imath}\right)$ is the characteristic function of the atom $\omega_{l}$. 
The proof of the main theorem (Theorem 3 below) will be based on the structure of isometries of spaces in class $\mathscr{S}$. Every bounded linear operator $A$ on $X \in \mathscr{S}$ can be represented by an operator matrix $\left(A_{i j}\right)$ where $A_{t l}: X_{j} \rightarrow X_{t}$. In [3] it was shown that every isometry $U$ of $X$ onto $X$ can be represented as $\left(U_{i \pi(l)}\right)$ where $U X_{\pi(i)}=X_{i}, U_{i \pi(i)}$ is unitary, and $\pi$ is a permutation of the positive integers (or of $\{1,2,3, \cdots, n\}$ in the case there are only finitely many of the $X_{i}$ 's).

ExAmple 6. If $X=l\left(p_{t}\right)$ and the $p_{i}$ 's are distinct then $U$ is an isometry of $X$ onto $X$ if and only if there exists a sequence of real numbers $\left\{\theta_{\imath}\right\}$ such that $(U x)(\kappa)=e^{i \theta_{\kappa}} X(\kappa)$ for each $\kappa$. In this case only the identity permutation is allowed. If $1<p_{t}=p<\infty, p \neq 2$, then $(U x)(\kappa)=e^{i \theta_{\kappa}} x(\pi(\kappa))$ where $\pi$ is any permutation of the positive integers.

We are now in a position to state and prove the main result.

Theorem 3. Let $X=\left(X,\left\{X_{i}\right\}, E, \mu\right) \in \mathscr{S} . U=\left\{U^{t} \mid t \in \mathbf{R}\right\}$ is a $\left(C_{0}\right)$ group of isometries on $X$ if and only if (i) for each $t \in \mathbf{R}, U^{t}$ has a diagonal operator matrix $\left(U_{\kappa \kappa}^{t}\right)$, and (ii) for each $\kappa,\left\{U_{\kappa \kappa}^{t}\right\}$ is a $\left(C_{0}\right)$ group of isometries acting on the Hilbert subspace $X_{\kappa}$.

Proof. (Necessity) For each $t \in \mathbf{R}, U^{t}$ is an isometry. Hence by Theorem 1 of [3], there is a permutation $\pi_{t}$ such that

$$
\left(U^{t} x\right)(i)=U_{t \pi_{t}(t)} x\left(\pi_{t}(i)\right) \text { for each } i,
$$

where $x=\Sigma x_{t} \in X$ and $U_{t \pi_{t}(t)}$ is a unitary map of $X_{\pi_{t}(t)}$ onto $X_{t}$.

Since $U=\left\{U^{t} \mid t \in \mathbf{R}\right\}$ is a group we have $U^{t} U^{s}=U^{t+s}$ for all $s, t \in \mathbf{R}$. Let $s, t$ be fixed and $x \in X$. Then

$$
\left(U^{s} U^{t} x\right)(i)=U_{t \pi_{s}(t)}^{s} U_{\pi_{s}(t) \pi_{\imath} \pi_{s}(t)}^{t} x\left(\pi_{t} \cdot \pi_{s}(i)\right)
$$

and

$$
\left(U^{s+t} x\right)(i)=U_{i \pi_{s+t}(t)}^{s+t} x\left(\pi_{s+t}(i)\right)
$$

Hence for every $x \in X$ we must have

$$
U_{l \pi_{s}(t)}^{s} U_{\pi_{s}(t) \pi_{t} \cdot \pi_{s}(t)}^{t} x\left(\pi_{t} \cdot \pi_{s}(i)\right)=U_{l \pi_{s+t}(l)} x\left(\pi_{s+t}(i)\right) .
$$

Let $i$ be given and suppose $\pi_{s+t}(i)=j$. Let $x$, denote a fixed nonzero 
element of the subspace $X_{j}$. Let $x(j)=x_{1}, x(\kappa)=0, \kappa \neq j$. Then with this $x$ we see that the right hand side of (3) is not zero. If $\pi_{s} \cdot \pi_{t}(i) \neq j$ the left hand side of (3) is zero. Hence $\pi_{s} \cdot \pi_{t}(i)=\pi_{s+t}(i)$ and since $i$ was arbitrary,

$$
\pi_{s} \cdot \pi_{t}=\pi_{s+t} .
$$

We now proceed to show that $\pi_{t}=$ identity for each $t$. We use the strong continuity of the group at zero. For each $x \in X$,

$$
U^{t} x \rightarrow x \quad \text { as } \quad t \rightarrow 0
$$

Let $i=1$ and let $x_{1}$ be a nonzero element of $X_{1}$. Set $x(i)=x_{1}$ if $i=1$, $x(i)=0$ if $i \neq 1$. Then from (5), there exists a neighborhood $V_{1}$ of $t=0$ such that $\nu\left(U^{t} x-x\right)<\left\|x_{1}\right\|$ for $t \in V_{1}$. Now

$$
\left(U^{t} x\right)(i)=U_{\imath \pi_{t}(t)}^{t} x\left(\pi_{t}(i)\right)= \begin{cases}0, & \pi_{t}(i) \neq 1 \\ U_{t 1}^{t} x_{1}, & \pi_{t}(i)=1 .\end{cases}
$$

Consequently, $\nu\left(U^{t} x-x\right) \geqq\left\|x_{1}\right\|=\nu(x)$ if $\pi_{t}(1) \neq 1$, for in this case, $U^{t} x-x=x_{1}+U_{11}^{t} x_{1}$. Thus $\pi_{t}(1)=1$ for all $t \in V_{1}$. In the same way, for given $N$, there exists a neighborhood $V_{N}$ of $t=0$ such that $\pi_{t}(\kappa)=\kappa$ for all $\kappa \leqq N$ and all $t \in V_{N}$. Now if $t_{0} \in \mathbf{R}$, there exists a positive integer $n$ such that $t_{0} / n \in V_{N}$. Therefore, $\pi_{t_{0} / n}(\kappa)=\kappa$ for all $\kappa \leqq N$ and by (4) $\pi_{t_{0}}=\pi_{n\left(t_{0} / n\right)}=\left[\pi_{t_{0} / n}\right]^{n}$. So that $\pi_{t_{0}}(\kappa)=\kappa$ for all $\kappa \leqq N$. Since $N$ was arbitrary we conclude that $\pi_{t}=$ identity permutation for each $t \in \mathbf{R}$.

This establishes that the operator matrix for $U^{t}$ is diagonal for each t. If follows from (3) that for each $i$,

$$
U_{i i}^{s} U_{i i}^{t} x(i)=U_{i i}^{s+t} x(i)
$$

for all $s, t \in \mathbf{R}$ and we have that each diagonal element is a member of a group of isometries acting on the corresponding Hilbert subspace. The continuity property of the diagonal element follows from the continuity of $U^{t}$.

For the converse, the only difficulty is in showing the continuity.

Let $x \in X$ and $\epsilon>0$ be given. Recall that $x=\Sigma x$, and $\nu(x)=$ $\mu\left[\left(\left\|x_{j}\right\|\right)\right]=\mu\left[\left(\left\|x_{1}\right\|,\left\|x_{2}\right\|, \cdots\right)\right]$. Hence there exists an $N$ such that

$$
\mu\left[\left(0,0, \cdots, 0,\left\|x_{N+1}\right\|,\left\|x_{N+2}\right\|, \cdots\right)\right]<\epsilon / 4 .
$$

Now, 
(7) $\left\{\begin{aligned} \nu\left(U^{t} x-x\right)= & \mu\left[\left(\left\|U_{i i}^{t} x(i)-x(i)\right\|\right)\right] \\ = & \mu\left[\left(\left\|U_{11}^{t} x(1)-x(1)\right\|, \cdots,\left\|U_{N N}^{t} x(N)-x(N)\right\|, 0,0, \cdots\right)\right] \\ & +\mu\left[\left(0,0, \cdots, 0,\left\|U_{N+1 N+1}^{t} x(N+1)-x(N+1)\right\|, \cdots\right)\right] .\end{aligned}\right.$

But $\left\|U_{\kappa \kappa}^{t} x(\kappa)-x(\kappa)\right\| \leqq 2\|x(\kappa)\|$ for all $t \in \mathbf{R}$ and every positive integer $\kappa$. Then from (7)

$$
\begin{aligned}
\nu\left(U^{t} x-x\right) \leqq & \mu\left[\left(\left\|U_{11}^{t} x(1)-x(1)\right\|, \cdots,\left\|U_{N N}^{t} x(N)-x(N)\right\|, 0,0, \cdots\right)\right] \\
& +2 \mu[(0,0,0, \cdots,\|x(N+1)\|, \cdots)] .
\end{aligned}
$$

Choose $V$ such that $t \in V$ implies $\left\|U_{i i}^{t} x(i)-x(i)\right\|<\epsilon / 4 M$ where $M=$ $\mu[(1, \cdots, 1,0,0, \cdots)]$, exactly $N$ 1's occurring inside the parentheses. Then $\nu\left(U^{t} x-x\right)<\epsilon$ for all $t \in V$. The proof is complete.

Remark 4. If the Hilbert subspaces $X_{t}$ are all one dimensional, then there exists a function $g: \mathbf{Z}^{+} \rightarrow \mathbf{R}$ such that $U^{t} x(\kappa)=$ $\exp \{\operatorname{itg}(\kappa)\} x(\kappa)$ for each $x \in X$ and $\kappa \in \mathbf{Z}^{+}$.

REMARK 5. If Theorem 3 is applied to the Orlicz space of Example 1 we find that every $\left(C_{0}\right)$ group of isometries on that space can be written as $U^{t}=\left[\begin{array}{cc}U_{11}^{t} & 0 \\ 0 & U_{22}^{t}\end{array}\right]$ where $\left\{U_{i i}^{t} \mid t \in \mathbf{R}\right\}$ is a $\left(C_{0}\right)$ group of isometries on $X_{t}$, $i=1,2$ respectively.

REMARK 6. Theorem 3 indirectly characterizes self conjugate operators (in the sense of Palmer [6]) on spaces of class $\mathscr{S}$. For example if $X=l\left(p_{\imath}\right)$, the self conjugate operators correspond to multiplications by real sequences. The bounded self conjugate operators are just the Hermitian operators and correspond to multiplication by bounded sequences.

\section{REFERENCES}

1. Z. Altshuler, P. G. Casazza, and Bor-Luh Lin, On symmetric basic sequences in Lorentz sequence spaces, Israel J. Math., 15 (1973), 140-155.

2. R. J. Fleming and J. E. Jamison, Hermitian and adjoint abelian operators on certain Banach spaces, Pacific J. Math., 52 (1974), 67-84.

3. - Isometries on certain Banach spaces, J. London Math. Soc., 9 (1975), 121-127.

4. J. A. Goldstein, Groups of isometries on Orlicz spaces, Pacific J. Math., 48 (1973), 387-393.

5. G. Lumer, Semı inner product spaces, Trans. Amer. Math. Soc., 100 (1961), 29-43.

6 T. W. Palmer, Unbounded normal operators on Banach spaces, Trans. Amer. Math. Soc., 133 (1968), 385-414.

7. K. Sundaresan, Uniform convexity of Banach spaces $l\left(p_{\imath}\right)$, Studia Math., 39 (1971), 227-231.

Received June 26, 1974.

MEMPhis State University 




\section{Pacific Journal of Mathematics}

Vol. 64, No. 1

May, 1976

Walter Allegretto, Nonoscillation theory of elliptic equations of order $2 n \ldots \ldots \quad 1$

Bruce Allem Anderson, Sequencings and starters.................. 17

Friedrich-Wilhelm Bauer, A shape theory with singular homology .......... 25

John Kelly Beem, Characterizing Finsler spaces which are

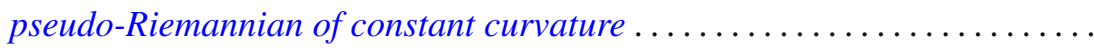

Dennis K. Burke and Ernest A. Michael, On certain point-countable

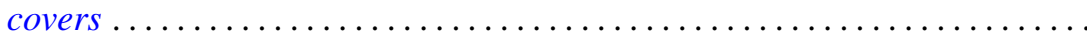

Robert Chen, A generalization of a theorem of Chacon ............... 93

Francis H. Clarke, On the inverse function theorem ................ 97

James Bryan Collier, The dual of a space with the Radon-Nikodým

property ....................................... 103

John E. Cruthirds, Infinite Galois theory for commutative rings ............ 107

Artatrana Dash, Joint essential spectra......................... 119

Robert M. DeVos, Subsequences and rearrangements of sequences in FK

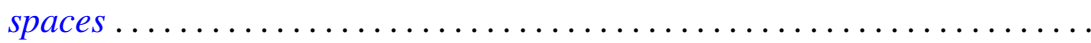

Geoffrey Fox and Pedro Morales, Non-Hausdorff multifunction generalization

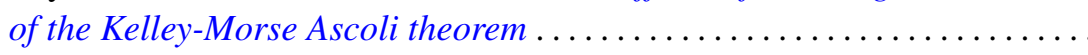

Richard Joseph Fleming, Jerome A. Goldstein and James E. Jamison, One

parameter groups of isometries on certain Banach spaces.............

Robert David Gulliver, II, Finiteness of the ramified set for branched

immersions of surfaces

Kenneth Hardy and István Juhász, Normality and the weak cb property ...... 167

C. A. Hayes, Derivation of the integrals of $L^{(q)}$-functions.

Frederic Timothy Howard, Roots of the Euler polynomials .

Robert Edward Jamison, II, Richard O'Brien and Peter Drummond Taylor, On

embedding a compact convex set into a locally convex topological vector space ....................................

Andrew Lelek, An example of a simple triod with surjective span smaller than span ...

Janet E. Mills, Certain congruences on orthodox semigroups

Donald J. Newman and A. R. Reddy, Rational approximation of $e^{-x}$ on the positive real axis.

John Robert Quine, Jr., Homotopies and intersection sequences ...

Nambury Sitarama Raju, Periodic Jacobi-Perron algorithms and fundamental units ....

Herbert Silverman, Convexity theorems for subclasses of univalent functions. . .

Charles Frederick Wells, Centralizers of transitive semigroup actions and endomorphisms of trees.........................

Volker Wrobel, Spectral approximation theorems in locally convex spaces ..................... 